\title{
GENERIC ABSOLUTENESS AND THE CONTINUUM
}

\section{Stevo Todorcevic}

Let $H_{\omega_{2}}$ denote the collection of all sets whose transitive closure has size at most $\aleph_{1}$. Thus, $\left(H_{\omega_{2}}, \in\right)$ is a natural model of ZFC minus the power-set axiom which correctly estimates many of the problems left open by the smaller and better understood structure $\left(H_{\omega_{1}}, \in\right)$ of hereditarily countable sets. One of such problems is, for example, the Continuum Hypothesis. It is largely for this reason that the structure $\left(H_{\omega_{2}}, \in\right)$ has recently received a considerable amount of study (see e.g. [15] and [16]). Recall the well-known Levy-Schoenfield absoluteness theorem $([10, \S 2])$ which states that for every $\Sigma_{0}$-sentence $\varphi(x, a)$ with one free variable $x$ and parameter $a$ from $H_{\omega_{2}}$, if there is an $x$ such that $\varphi(x, a)$ holds then there is such an $x$ in $H_{\omega_{2}}$, or in other words,

$$
\left(H_{\omega_{2}}, \in\right) \prec_{1}(V, \in) \text {. }
$$

Strictly speaking, what is usually called the Levy-Schoenfield absoluteness theorem is a bit stronger result than this, but this is the form of their absoluteness theorem that allows a variation of interest to us here. The generic absoluteness considered in this paper is a natural strengthening of (1) where the universe $V$ is replaced by one of its boolean-valued extensions $V^{\mathcal{B}}$, i.e. the statement of the form

$$
\left(H_{\omega_{2}}, \in\right) \prec_{1}\left(V^{\mathcal{B}}, \in\right)
$$

for a suitably chosen boolean-valued extension $V^{\mathcal{B}}$. This sort of generic absoluteness has apparently been first considered by J. Stavi (see [11]) and then by J. Bagaria [4] who has also observed that any of the 'Bounded Forcing Axioms' introduced by M.Goldstern and S.Shelah [9] is equivalent to the corresponding generic absoluteness statement for the structure $\left(H_{\omega_{2}}, \in\right)$. The most prominent such statement (besides of course Martin's axiom; see [6]) is the Bounded Martin's Maximum which asserts (2) for any boolean-valued extension $V^{\mathcal{B}}$ which preserves stationary subsets of $\omega_{1}$ (see [1]-[4], [6]-[9], [11]-[12], [14]-[16]). The purpose of this note is to answer the natural question (appearing explicitely or implicitly in some of the listed papers) which asks whether any of the standard forms of the generic absoluteness (2) discussed above decides the size of the continuum.

Theorem 1. Assume generic absoluteness (2) for boolean-valued extensions which preserve stationary subsets of $\omega_{1}$. Then there is a well ordering of the continuum of length $\omega_{2}$ which is definable in the structure $\left(H_{\omega_{2}}, \in\right)$.

Received May 6, 2002. 
Remark 2. It should be noted that prior to our work, substantial and quite inspiring advances towards Theorem 1 had already been made. For example, W.H. Woodin $[16, \S 10.3]$ proved Theorem 1 under the additional assumption of the existence of a measurable cardinal and D. Aspero [2] proved the weaker conclusion in Theorem 1 to the effect that there is $\mathcal{F} \subseteq \omega^{\omega}$ of size $\aleph_{2}$ which is cofinal in the ordering of eventual dominance of $\omega^{\omega}$.

We start proving Theorem 1 by fixing the only parameter of our definition, a one-to-one sequence $r_{\xi}\left(\xi<\omega_{1}\right)$ of elements of the Cantor set $2^{\omega}$. This allows us to associate to every countable set of ordinals $X$, the real

$$
r_{X}=r_{\mathrm{otp}(X)} \text {. }
$$

(See [13] for a more precise functor $X \rightarrow r_{\mathrm{otp}(X)}$ which can also be used in the definitions that follow.) For a pair $x$ and $y$ of distinct members of $2^{\omega}$, set

$$
\Delta(x, y)=\min \{n<\omega: x(n) \neq y(n)\} .
$$

Note that for three distinct members $x, y$ and $z$ of $2^{\omega}$, the set

$$
\Delta(x, y, z)=\{\Delta(x, y), \Delta(y, z), \Delta(x, z)\}
$$

has exactly two elements.

Definition 3. Let $\theta_{A C}$ denote the statement that for every $S \subseteq \omega_{1}$ there exist ordinals $\gamma>\beta>\alpha \geq \omega_{1}$ and an increasing continuous decomposition

$$
\gamma=\bigcup_{\nu<\omega_{1}} N_{\nu}
$$

of the ordinal $\gamma$ into countable sets such that for all $\nu<\omega_{1}$,

$$
N_{\nu} \cap \omega_{1} \in S \text { iff } \Delta\left(r_{N_{\nu} \cap \alpha}, r_{N_{\nu} \cap \beta}\right)=\max \Delta\left(r_{N_{\nu} \cap \alpha}, r_{N_{\nu} \cap \beta}, r_{N_{\nu}}\right) .
$$

For a given $S \subseteq \omega_{1}$ we let $\theta_{A C}^{1}\left(S,\left\{r_{\xi}: \xi<\omega_{1}\right\}\right)$ denote the $\Sigma_{1}$-sentence of $\left(H_{\omega_{2}}, \in\right)$ asserting the existence of $\gamma>\beta>\alpha \geq \omega_{1}$ and the decomposition $N_{\nu}\left(\nu<\omega_{1}\right)$ of $\gamma$ satisfying the equivalence (3) for all $\nu<\omega_{1}$.

Theorem 1 follows from the following more informative result.

Theorem 4. For every $S \subseteq \omega_{1}$ there is a boolean-valued extension which preserves stationary subsets of $\omega_{1}$ and satisfies $\theta_{A C}^{1}\left(S,\left\{r_{\xi}: \xi<\omega_{1}\right\}\right)$.

Corollary 5. The generic absoluteness (2) for boolean extensions which preserve stationary subsets of $\omega_{1}$ implies $\theta_{A C}$.

We need a few definitions, so let $\lambda=2^{2^{\aleph_{1}}}$.

For $\omega_{1} \leq \alpha<\beta<\gamma \leq \lambda^{+}$and $P \in\{\min , \max \}$, set

$$
\mathcal{S}_{\alpha \beta \gamma}^{P}=\left\{N \in[\gamma]^{\omega}: \Delta\left(r_{N \cap \alpha}, r_{N \cap \beta}\right)=P \Delta\left(r_{N \cap \alpha}, r_{N \cap \beta}, r_{N}\right)\right\} .
$$

For $A \subseteq \omega_{1}$ and $\alpha, \beta, \gamma$ and $P$ as above, set

$$
\mathcal{S}_{\alpha \beta \gamma}^{P}(A)=\left\{N \in \mathcal{S}_{\alpha \beta \gamma}^{P}: N \cap \omega_{1} \in A\right\} .
$$


For $\omega_{1} \leq \alpha<\beta<\lambda^{+}, A \subseteq \omega_{1}$ and $P \in\{\min , \max \}$, set

$$
\Gamma_{\alpha \beta}^{P}(A)=\left\{\gamma<\lambda^{+}: \mathcal{S}_{\alpha \beta \gamma}^{P}(A) \text { is stationary }\right\} .
$$

Lemma 6. There exist $\omega_{1} \leq \alpha<\beta<\lambda^{+}$, such that $\Gamma_{\alpha \beta}^{P}(A)$ is stationary for all stationary $A \subseteq \omega_{1}$ and $P \in\{\min , \max \}$.

Proof. Otherwise, for each $\omega_{1} \leq \alpha<\beta<\lambda^{+}$, we can choose a stationary $A(\alpha, \beta) \subseteq \omega_{1}$ and $P(\alpha, \beta) \in\{\min , \max \}$ such that

$$
D(\alpha, \beta)=\left\{\gamma<\lambda^{+}: \mathcal{S}_{\alpha \beta \gamma}^{P(\alpha, \beta)}(A(\alpha, \beta)) \text { is nonstationary }\right\}
$$

contains a closed and unbounded subset of $\lambda^{+}$. It follows that there is a single closed and unbounded set $D \subseteq \lambda^{+}$such that for all $\alpha<\beta$ in $D$,

$$
D \backslash(\beta+1) \subseteq D(\alpha, \beta) .
$$

Applying $\lambda^{+} \rightarrow(\omega+2)_{2^{\aleph_{1}}}^{2}$ to the coloring of $(\alpha, \beta) \mapsto(A(\alpha, \beta), P(\alpha, \beta))$, we can find $E \subseteq D$ of order-type $\omega+2$, a stationary set $A \subseteq \omega_{1}$, and $P \in\{\min , \max \}$ such that $A(\alpha, \beta)=A$ and $P(\alpha, \beta)=P$ for all $\alpha<\beta$ in $E$. It follows that

$$
\mathcal{S}_{\alpha \beta \gamma}^{P}(A) \text { is nonstationary for all } \alpha<\beta<\gamma \text { in } E \text {. }
$$

Let $\kappa$ be a large enough regular cardinal and $M_{\nu}\left(\nu<\omega_{1}\right)$ a continuous $\in$ chain of countable elementary submodels of $\left(H_{\kappa}, \in\right)$ containing all the objects accumulated so far. Let

$$
N_{\nu}=M_{\nu} \cap \lambda^{+},\left(\nu<\omega_{1}\right),
$$

and

$$
A_{0}=\left\{\nu \in A: N_{\nu} \cap \omega_{1}=\nu\right\} .
$$

Then $A_{0}$ is stationary as $A \backslash A_{0}$ is not. Note that for $\nu<\omega_{1}$ and $\alpha<\beta<\gamma$ from $E$, the set $\mathcal{S}_{\alpha \beta \gamma}^{P}(A)$ belongs to $M_{\nu}$, so by (4)

$$
N_{\nu} \cap \gamma=M_{\nu} \cap \gamma \notin \mathcal{S}_{\alpha \beta \gamma}^{P}(A) .
$$

It follows that:

$$
\begin{gathered}
\Delta\left(r_{N_{\nu} \cap \alpha}, r_{N_{\nu} \cap \beta}\right)=\bar{P} \Delta\left(r_{N_{\nu} \cap \alpha}, r_{N_{\nu} \cap \beta}, r_{N_{\nu} \cap \gamma}\right) \text { for all } \\
\nu \in A_{0} \text { and } \alpha<\beta<\gamma \text { from } E
\end{gathered}
$$

where $\bar{P}=\min$ if $P=\max$ and $\bar{P}=\max$ if $P=\min$.

Case 1. $\bar{P}=\min$. For $\alpha<\beta$ in $E$ and $k \in \omega$, set

$$
A_{\alpha \beta}[\geq k]=\left\{\nu \in A_{0}: \Delta\left(r_{N_{\nu} \cap \alpha}, r_{N_{\nu} \cap \beta}\right) \geq k\right\} .
$$

For $\alpha<\beta$ in $E$, set

$$
k_{\alpha \beta}=\max \left\{k \in \omega: A \backslash A_{\alpha \beta}[\geq k] \text { is nonstationary }\right\} .
$$

Fix $\alpha<\beta<\gamma<\delta$ in $E$. Consider $\nu \in A_{\alpha \beta}\left[\geq k_{\alpha \beta}\right]$. Applying (5) to triples $\alpha<\beta<\gamma$ and $\alpha<\beta<\delta$, we get that 


$$
\begin{aligned}
& \Delta\left(r_{N_{\nu} \cap \alpha}, r_{N_{\nu} \cap \beta}\right)<\Delta\left(r_{N_{\nu} \cap \beta}, r_{N_{\nu \cap \gamma}}\right), \text { and } \\
& \Delta\left(r_{N_{\nu} \cap \alpha}, r_{N_{\nu} \cap \beta}\right)<\Delta\left(r_{N_{\nu} \cap \beta}, r_{N_{\nu} \cap \delta}\right) .
\end{aligned}
$$

Note that this gives the equalities

$$
r_{N_{\nu} \cap \gamma}\left\lceil n=r_{N_{\nu} \cap \beta}\left\lceil n=r_{N_{\nu} \cap \delta}\lceil n\right.\right.
$$

for $n=\Delta\left(r_{N_{\nu} \cap \alpha}, r_{N_{\nu} \cap \beta}\right)+1$. It follows that,

$$
k_{\alpha \beta} \leq \Delta\left(r_{N_{\nu \cap \alpha}}, r_{N_{\nu} \cap \beta}\right)<\Delta\left(r_{N_{\nu \cap \gamma}}, r_{N_{\nu} \cap \delta}\right) .
$$

Since $\nu$ was chosen to be an arbitrary member of $A_{\alpha \beta}\left[\geq k_{\alpha \beta}\right]$, we conclude that the set

$$
A \backslash A_{\gamma \delta}\left[\geq k_{\alpha \beta}+1\right]
$$

is nonstationary. Hence $k_{\gamma \delta} \geq k_{\alpha \beta}+1$. This shows that

$$
k_{\alpha \beta}<k_{\gamma \delta} \text { for all } \alpha<\beta<\gamma<\delta \text { from } E,
$$

and this is in direct contradiction with the fact that $E$ has order-type $\omega+2$.

Case 2. $\bar{P}=\max$. Fix $\alpha<\beta<\gamma<\delta$ in $E$ and consider an arbitrary $\nu \in A_{\gamma \delta}[\geq$ $k_{\gamma \delta}$ ]. Applying (5) to triples $\alpha<\gamma<\delta$ and $\beta<\gamma<\delta$, we get that

$$
\begin{aligned}
& \Delta\left(r_{N_{\nu} \cap \alpha}, r_{N_{\nu} \cap \gamma}\right)>\Delta\left(r_{N_{\nu} \cap \gamma}, r_{N_{\nu \cap \delta}}\right), \text { and } \\
& \Delta\left(r_{N_{\nu} \cap \beta}, r_{N_{\nu} \cap \gamma}\right)>\Delta\left(r_{N_{\nu} \cap \gamma}, r_{N_{\nu} \cap \delta}\right) .
\end{aligned}
$$

This gives that

$$
r_{N_{\nu} \cap \alpha}\left\lceil n=r_{N_{\nu} \cap \gamma}\left\lceil n=r_{N_{\nu} \cap \beta}\lceil n\right.\right.
$$

for $n=\Delta\left(r_{N_{\nu} \cap \gamma}, r_{N_{\nu} \cap \delta}\right)+1$. It follows that,

$$
k_{\gamma \delta} \leq \Delta\left(r_{N_{\nu \cap \gamma}}, r_{N_{\nu} \cap \delta}\right)<\Delta\left(r_{N_{\nu \cap \alpha}}, r_{N_{\nu} \cap \beta}\right) .
$$

Since $\nu$ is an arbitrary member of $A_{\gamma \delta}\left[\geq k_{\gamma \delta}\right]$, we conclude that the set

$$
A \backslash A_{\alpha \beta}\left[\geq k_{\gamma \delta}+1\right]
$$

is nonstationary. Hence $k_{\alpha \beta} \geq k_{\gamma \delta}+1$. This shows that

$$
k_{\alpha \beta}>k_{\gamma \delta} \text { for all } \alpha<\beta<\gamma<\delta \text { from } E,
$$

and this can happen only if the set $E$ is finite, a contradiction. The case-analysis shows that our initial assumption that the conclusion of Lemma 6 is false, leads to contradictions finishing thus the proof.

Fix $\omega_{1} \leq \alpha<\beta<\lambda^{+}$satisfying the conclusion of Lemma 6.

Lemma 7. For every stationary set $A \subseteq \omega_{1}$ and $P \in\{\min , \max \}$, the set

$$
\begin{gathered}
\mathcal{S}_{\alpha \beta}^{P}(A)=\left\{N \in\left[\lambda^{+}\right]^{\omega}: N \cap \omega_{1} \in A \& \Delta\left(r_{N_{\nu} \cap \alpha}, r_{N_{\nu} \cap \beta}\right)=\right. \\
\left.=P \Delta\left(r_{N_{\nu} \cap \alpha}, r_{N_{\nu} \cap \beta}, r_{N}\right)\right\}
\end{gathered}
$$

is stationary. 
Proof. Consider an $f:\left(\lambda^{+}\right)^{<\omega} \rightarrow \lambda^{+}$. We need to find $N \in \mathcal{S}_{\alpha \beta}^{P}(A)$ such that $f^{\prime \prime}(N)^{<\omega} \subseteq N$. Since $\alpha$ and $\beta$ satisfy the conclusion of Lemma 6 , the set $\Gamma_{\alpha \beta}^{P}(A)$ is a stationary subset of $\lambda^{+}$, so there is $\gamma \in \Gamma_{\alpha \beta}^{P}(A)$ such that $f^{\prime \prime} \gamma^{<\omega} \subseteq \gamma$. Then $\mathcal{S}_{\alpha \beta \gamma}^{P}(A)$ is stationary so applying this to the restriction $g=f \uparrow \gamma^{<\omega}$ we find $N \in \mathcal{S}_{\alpha \beta \gamma}^{P}(A)$ such that $g^{\prime \prime} N^{<\omega} \subseteq N$. Note that $N \in \mathcal{S}_{\alpha \beta}^{P}(A)$ and that $g^{\prime \prime} N^{<\omega}=f^{\prime \prime} N^{<\omega}$. This finishes the proof.

We are now ready to start the proof of Theorem 4 .

Proof of Theorem 4. Fix a subset $S$ of $\omega_{1}$. Let $\mathcal{P}$ be the poset of all countable increasing continuous transfinite sequences of the form $p=\left\langle N_{\nu}^{p}: \nu \leq \nu_{p}\right\rangle$ such that for all $\nu \leq \nu_{p}$,

$$
N_{\nu}^{p} \cap \omega_{1} \in S \text { iff } \Delta\left(r_{N_{\nu}^{p} \cap \alpha}, r_{N_{\nu}^{p} \cap \beta}\right)=\max \Delta\left(r_{N_{\nu}^{p} \cap \alpha}, r_{N_{\nu}^{p} \cap \beta}, r_{N_{\nu}^{p}}\right) .
$$

Clearly, it suffices to show that $\mathcal{P}$ preserves stationary subsets of $\omega_{1}$. So, consider a stationary subset $A$ of $\omega_{1}, p \in \mathcal{P}$, and a $\mathcal{P}$-term $\tau$ for a closed and unbounded subset of $\omega_{1}$. Let $\kappa$ be a large enough regular cardinal so that the structure $\left(H_{\kappa}, \in\right)$ contains all the objects accumulated so far. Suppose first that $A \cap S$ is stationary. By Lemma 7, the set $\mathcal{S}_{\alpha \beta}^{\max }(A \cap S)$ is stationary, so we can find a countable elementary submodel $M$ of $\left(H_{\kappa}, \in\right)$ containing all the relevant objects such that

$$
M \cap \lambda^{+} \in \mathcal{S}_{\alpha \beta}^{\max }(A \cap S)
$$

Working in $M$ we build a decreasing sequence $p=p_{0} \geq p_{1} \geq \cdots \geq p_{n} \geq \cdots$ of elements of $\mathcal{P} \cap M$ and a sequence $\xi_{n}(n<\omega)$ of ordinals from $M \cap \omega_{1}$ such that:

(a) $M \cap \lambda^{+}=\bigcup_{n<\omega} \bigcup_{\nu \leq \nu_{p_{n}}} N_{\nu}^{p_{n}}$

(b) $p_{n}$ forces that $\xi_{n}$ belongs to $\tau$,

(c) $\sup _{n<\omega} \xi_{n}=M \cap \omega_{1}$.

Let $\nu_{q}=\sup _{n<\omega} \nu_{p_{n}}$ and

$$
q=\left(\bigcup_{n<\omega} p_{n}\right) \cup\left\{\left\langle\nu_{q}, M \cap \lambda^{+}\right\rangle\right\} .
$$

Then $q \in \mathcal{P}, q \leq p$ and $q$ forces that $M \cap \omega_{1}$ belongs to the intersection of $\tau$ and $A$. The case when $A \backslash S$ is stationary is considered similarly using Lemma 7 for $A \backslash S$ and $P=\min$, i.e., the fact that $\mathcal{S}_{\alpha \beta}^{\min }(A \backslash S)$ is stationary. This finishes the proof of Theorem 4 .

We finish this note with the following observation which shows that $\theta_{A C}$ not only gives an upper bound on the size of the continuum but also the exact value.

Theorem 8. $\theta_{A C}$ implies $2^{\aleph_{0}}=2^{\aleph_{1}}=\aleph_{2}$.

Proof. It remains to show that $\theta_{A C}$ implies $2^{\aleph_{0}}=2^{\aleph_{1}}$. This will be done by showing that $\theta_{A C}$ violates the weak-diamond principle of Devlin and Shelah [5]. Define $F: 2^{<\omega_{1}} \rightarrow 2$ by letting $F(f)=0$ iff $f$ codes (in the usual way) a well 
ordering $<_{f}$ of its domain $\nu$ which has to be a countable limit ordinal bigger than 0 such that if we let

$$
\begin{aligned}
\alpha_{f} & =\operatorname{otp}\left(\left\{\xi<\nu: \xi<_{f} 0\right\},<_{f}\right), \\
\beta_{f} & =\operatorname{otp}\left(\left\{\xi<\nu: \xi<_{f} 1\right\},<_{f}\right), \\
\gamma_{f} & =\operatorname{otp}\left(\nu,<_{f}\right),
\end{aligned}
$$

then $\alpha_{f}<\beta_{f}<\gamma_{f}$ and

$$
\Delta\left(r_{\alpha_{f}}, r_{\beta_{f}}\right)=\max \Delta\left(r_{\alpha_{f}}, r_{\beta_{f}}, r_{\gamma_{f}}\right) .
$$

If weak-diamond holds it would apply to $F$ giving us $g \in 2^{\omega_{1}}$ such that for every $f \in 2^{\omega_{1}}$, the set

$$
\left\{\nu<\omega_{1}: F(f\lceil\nu)=g(\nu)\}\right.
$$

is a stationary subset of $\omega_{1}$. Let $S$ be equal to $g^{-1}(1)$. Applying $\theta_{A C}$ to $S$ we get $\omega_{1} \leq \alpha<\beta<\gamma<\omega_{2}$ and an increasing continuous decomposition

$$
\gamma=\bigcup_{\nu<\omega_{1}} N_{\nu}
$$

of the ordinal $\gamma$ into countable subsets such that for all $\nu<\omega_{1}$,

$$
N_{\nu} \cap \omega_{1} \in S \text { iff } \Delta\left(r_{N_{\nu} \cap \alpha}, r_{N_{\nu} \cap \beta}\right)=\max \Delta\left(r_{N_{\nu} \cap \alpha}, r_{N_{\nu} \cap \beta}, r_{N_{\nu}}\right) .
$$

Then we can find $f \in 2^{\omega_{1}}$ which codes a well-ordering $<_{f}$ of $\omega_{1}$ of order type $\gamma$ so that for almost all countable limit ordinals $\nu$,

$$
\begin{aligned}
\alpha_{f \nmid \nu} & =\operatorname{otp}\left(N_{\nu} \cap \alpha\right), \\
\beta_{f \uparrow \nu} & =\operatorname{otp}\left(N_{\nu} \cap \beta\right), \\
\gamma_{f \nmid \nu} & =\operatorname{otp}\left(N_{\nu}\right) .
\end{aligned}
$$

It follows that for almost all $\nu<\omega_{1}$,

$$
\nu \in S \text { iff } \Delta\left(r_{\alpha_{f \uparrow \nu}}, r_{\beta_{f \nmid \nu}}\right)=\max \Delta\left(r_{\alpha_{f \nmid \nu}}, r_{\beta_{f \nmid \nu}}, r_{\gamma_{f \uparrow \nu}}\right) .
$$

Let $A=\left\{\nu<\omega_{1}: F(f \uparrow \nu)=g(\nu)\right\}$. Then by the choice of $g$, the set $A$ is stationary. If $A \cap S$ is stationary, we can find $\nu \in A \cap S$ for which the equivalence (8) is true, i.e., such that

$$
\Delta\left(r_{\alpha_{f \uparrow \nu}}, r_{\beta_{f \uparrow \nu}}\right)=\max \Delta\left(r_{\alpha_{f \uparrow \nu}}, r_{\beta_{f \uparrow \nu}}, r_{\gamma_{f \uparrow \nu}}\right) .
$$

Going back to the definition of $F$ we see that $F(f \uparrow \nu)=0$, and therefore, $g(\nu)=0$ which means that $\nu \notin S$, a contradiction. If $A \backslash S$ is stationary we chose $\nu \in A \backslash S$ satisfying the equivalence (8), i.e., such that

$$
\Delta\left(r_{\alpha_{f \nmid \nu}}, r_{\beta_{f \uparrow \nu}}\right)=\min \Delta\left(r_{\alpha_{f \uparrow \nu}}, r_{\beta_{f \nmid \nu}}, r_{\gamma_{f \uparrow \nu}}\right) .
$$

Applying the definition of $F$, we see that $F(f\lceil\nu)=1$, and therefore $g(\nu)=1$ which means $\nu \in S$, a contradiction. This finishes the proof of Theorem 8 . 


\section{References}

[1] D. Asperó, Bounded forcing axioms and the continuum, preprint, 2000.

[2] _ Bounded Martin's Maximum, d and c, preprint, 2002.

[3] D. Asperó, J. Bagaria, Bounded forcing axioms and the continuum, Ann. Pure, Appl. Logic. 109 (2001), 179-203.

[4] J. Bagaria, Bounded forcing axioms as principles of generic absoluteness, Arch. Math. Logic 39 (2000), 393-401.

[5] K. J. Devlin, S. Shelah, A weak version of diamond which follows from $2^{\aleph_{0}}<2^{\aleph_{1}}$, Israel J. Math. 29 (1978), 239-247.

[6] M. Foreman, M. Magidor, S. Shelah, Martin's maximum, saturated ideals and non regular ultrafilters. I. Ann. of Math. (2) 127 (1998), 1-47.

[7] D. H. Fremlin, Consequences of Martin's axiom, Cambridge Tracts in Mathematics, 84. Cambridge University Press, Cambridge, 1984.

[8] S. Fuchino, On potential embedding and versions of Martin's axiom, Notre Dame J. Formal Logic 33 (1992), 481-492.

[9] M. Goldstern, S. Shelah, The bounded proper forcing axiom, J. Symbolic Logic 60 (1995), $58-73$.

[10] T. Jech, Set Theory. Pure and Applied Mathematics, Academic Press, New York-London, 1978.

[11] T. Miyamoto, Localized reflecting cardinals and weak fragments of PFA, preprint, 1996.

[12] J. Stavi, J. Väänänen, Reflection principles for the continuum. preprint, 1981.

[13] S. Todorcevic, Partitioning pairs of countable sets, Proc. Amer. Math. Soc. 111 (1991), 841-844.

[14] _ Comparing the continuum with the first two uncountable cardinals, Logic and scientific methods (Florence, 1995), 145-155, Synthese Lib., 259, Kluwer Acad. Publ., Dordrecht, 1997.

[15] _ Localized reflection and fragments of PFA, DIMACS Series in Discr. Math. and Theoret. Comp. Sci. 58 (2002), 135-148.

[16] H. Woodin, The axiom of determinacy, forcing axioms, and the nonstationary ideal, de Gruyter Series in Logic and its Applications, 1. Walter de Gruyter \& Co., Berlin, 1999.

[17] - The Continuum Hypothesis. I, II. Notices Amer. Math. Soc. 48 (2001), 567-576, 681-690.

Université de Paris 7 - C.N.R.S., UMR 7056, 2 Place Jussieu - Case 7012, 75251

Paris Cedex 05, France.

E-mail address: stevo@math.jussieu.fr 